\title{
Spectral Index in Curvaton Scenario
}

\author{
Qing-Guo Huang \\ School of physics, Korea Institute for Advanced Study, \\ 20\%-43, Cheongryangri-Dong, Dongdaemun-Gu, \\ Seoul 130-722, Korea \\ huangqg@kias.re.kr
}

\begin{abstract}
A red tilted primordial power spectrum is preferred by WMAP five-year data and a large positive local-type non-Gaussianity $f_{N L}$ might be observed as well. In this short note we find that a red tilted and large non-Gaussian primordial power spectrum cannot be naturally obtained in curvaton model, because $f_{N L}$ is related to the initial condition of inflation.
\end{abstract}


Inflation [1] provides an elegant mechanism to solve many puzzles in the Hot Big Bang model. The wrinkles in the cosmic microwave background radiation and the largescale structure of the Universe are seeded by the quantum fluctuations generated during inflation [2]. The shape of the primordial quantum fluctuations is characterized by its amplitude $P_{\zeta}$ and tilt $n_{s}$ which can be measured by experiments. WMAP five-year data [3] combined with the distance measurements from the Type Ia supernovae (SN) and the Baryon Acoustic Oscillations (BAO) in the distribution of galaxies indicates

$$
\begin{aligned}
P_{\zeta, o b s} & =2.457_{-0.093}^{+0.092} \times 10^{-9} \\
n_{s} & =0.960_{-0.013}^{+0.014}
\end{aligned}
$$

Gravitational wave perturbations are also generated during inflation and its amplitude $P_{T}$ is only determined by the inflation scale. For convenience, we define a new quantity, named tensor-scalar ratio $r=P_{T} / P_{\zeta}$, to measure the amplitude of gravitational wave perturbations. The primordial gravitational wave perturbation has not been detected. Present limit on the tensor-scalar ratio is $r<0.20$ (95\% CL). The blue tilted primordial power spectrum $\left(n_{s}>1\right)$ is disfavored even when gravitational waves are included.

The non-Gaussianity is characterized by the non-Gaussianity parameter $f_{N L}$ which is defined as follows

$$
\Phi(\mathbf{x})=\Phi_{L}(\mathbf{x})+f_{N L}\left[\Phi_{L}^{2}(\mathbf{x})-\left\langle\Phi_{L}^{2}(\mathbf{x})\right\rangle\right],
$$

where $\Phi_{L}(\mathbf{x})$ denotes the linear Gaussian part of the perturbation in real space. The simplest model of inflation predicts a closely Gaussian distribution of primordial fluctuations $[4,5]$, namely $\left|f_{N L}\right|<\mathcal{O}(1)$. The most general density perturbation is a superposition of an isocurvature density perturbation and an adiabatic density perturbation. We introduce a new parameter $\alpha_{-1}=f_{\text {iso }}^{2} /\left(1+f_{\text {iso }}^{2}\right)$ to measure the isocurvature density perturbation, where $f_{i s o}$ is the ratio of the isocurvature and adiabatic amplitudes at the pivot scale. A Gaussian and adiabatic power spectrum of primordial perturbation is still consistent with WMAP five-year data:

$$
\begin{gathered}
-9<f_{N L}^{\text {local }}<111 \text { and }-151<f_{N L}^{\text {equil }}<253 \quad(95 \% \mathrm{CL}), \\
\alpha_{-1}<0.0037(95 \% \mathrm{CL}),
\end{gathered}
$$

where "local" and "equil" denote the shapes of the non-Gaussianity. In [6] the authors reported that a positive large non-Gaussianity

$$
27<f_{N L}^{\text {local }}<147
$$

is detected at $95 \%$ C.L.. A large non-Gaussianity is not a conclusive result from experiments, but it is still worthy for us to discussing the theoretical probabilities of the 
large non-Gaussianity. If it is confirmed by the forthcoming cosmological experiments, it strongly shows up some very important new physics of the early Universe.

An attractive model for a large positive local-type non-Gaussianity is curvaton model $[7,8]$ in which the primordial power spectrum is generated by a light scalar field, called curvaton $\sigma$, but not the inflaton $\phi$, even though the dynamics of inflation is governed by the inflaton. Recently many issues about curvaton model were discussed in [9-12]. In [9] we considered the case in which the Hubble parameter is roughly a constant during inflation and found that $f_{N L}$ is bounded by the tensor-scalar ratio $r$ from above. Or equivalently a large non-Gaussianity gives a lower bound on the amplitude of the tensor perturbation in curvaton scenario. In [3] the authors pointed out that a large positive $f_{N L}$ cannot be obtained by considering the bound on the isocurvature perturbation $\alpha_{-1}<0.0037$ in curvaton model. However if the cold dark matter was produced after the curvaton decays completely, the curvaton model is free from the constraint of isocurvature perturbation [12]. Some other related topics on the large non-Gaussianity are discussed in [13] recently.

The spectral index of primordial power spectrum in curvaton scenario is given by

$$
n_{s}=1-2 \epsilon+2 \eta_{\sigma \sigma}
$$

where $\eta_{\sigma \sigma}=\frac{1}{3 H^{2}} \frac{d^{2} V(\sigma)}{d \sigma^{2}}$ and

$$
\epsilon \equiv-\dot{H} / H^{2}
$$

denotes how fast the Hubble parameter varies during inflation. Usually in curvaton model we assume that the mass of curvaton $\frac{d^{2} V(\sigma)}{d \sigma^{2}}$ is much smaller than the Hubble parameter during inflation. Therefore $n_{s} \simeq 1-2 \epsilon$. For $n_{s}=0.96, \epsilon \simeq 0.02$. Such a large value of $\epsilon$ implies the variation of inflaton is larger than Planck scale, which might be inconsistent with string theory [14-17]. Usually a small value of $\epsilon$ and a closely scale-invariant power spectrum are expected in curvaton scenario. However, maybe the effective tran-Planckian excursion of inflation can be achieved in string landscape [18] or the monodromies [19].

In this short note we will extend our previous work [9] to more general cases and investigate whether a red tilted primordial power spectrum is naturally compatible with a large positive $f_{N L}$ in curvaton model.

Let us consider a simple curvaton model with potential

$$
V(\phi, \sigma)=V(\phi)+\frac{1}{2} m^{2} \sigma^{2}
$$

where $V(\phi) \gg \frac{1}{2} m^{2} \sigma^{2}, \phi$ and $\sigma$ denote the inflaton and curvaton respectively. The slowroll equations of motion are obtained by neglecting the kinetic term and the energy density of curvaton in the Friedmann equation, and the second time derivative in the inflaton field 
equation, namely

$$
\begin{aligned}
H^{2} & =\frac{V(\phi)}{3 M_{p}^{2}}, \\
3 H \dot{\phi} & =-V^{\prime}(\phi),
\end{aligned}
$$

where $V^{\prime}(\phi)=d V(\phi) / d \phi$ and $M_{p}$ is the reduced Planck scale. Once inflation is over, the energy density of inflaton is converted into radiation and $H^{2}$ goes like $a^{-4}$. The value of curvaton field, denoted as $\sigma_{*}$, does not change until the Hubble parameter becomes the same order of curvaton mass. After that curvaton oscillates around its minimum $\sigma=0$ and its energy density decreases as $a^{-3}$. Once the Hubble parameter goes to the same order of the curvaton decay rate $\Gamma_{\sigma}$, the curvaton energy is converted into radiations. Before primordial nucleosynthesis, the curvaton field is supposed to completely decay into radiation and thus the perturbations in the curvaton field are converted into curvature perturbations. The amplitude of the perturbations caused by curvaton is given in [8] by

$$
P_{\zeta_{\sigma}}^{\frac{1}{2}}=\frac{1}{3 \pi} \Omega_{\sigma, D} \frac{H_{*}}{\sigma_{*}}
$$

where

$$
\Omega_{\sigma, D} \equiv\left(\frac{\rho_{\sigma}}{\rho_{t o t}}\right)_{D}
$$

is the fraction of curvaton energy density in the energy budget at the time of $H=\Gamma_{\sigma}$. Here the subscript * denotes that the quantities are evaluated at horizon exit during inflation. A large positive non-Gaussianity is obtained only when $\Omega_{\sigma, D} \ll 1$ and $f_{N L}$ is given by

$$
f_{N L}=\frac{2}{3}-\frac{5}{6} \Omega_{\sigma, D}+\frac{5}{4 \Omega_{\sigma, D}} \simeq \frac{5}{4 \Omega_{\sigma, D}} .
$$

In the case of $\Omega_{\sigma, D} \ll 1$ the Universe is dominated by radiation before the time of $H=\Gamma_{\sigma}$. In [8] the value of $\Omega_{\sigma, D}$ is estimated as

$$
\Omega_{\sigma, D} \simeq \frac{\sigma_{*}^{2}}{6 M_{p}^{2}}\left(\frac{m}{\Gamma_{\sigma}}\right)^{\frac{1}{2}} .
$$

Keeping $m$ and $\Gamma_{\sigma}$ fixed, $f_{N L} \propto M_{p}^{2} / \sigma_{*}^{2}$. In the literatures $\sigma_{*}$ is treated as a free parameter and then a large $f_{N L}$ is naturally expected for $\sigma_{*}<M_{p}$. However this treatment seems too naive. The curvaton mass is much smaller than the Hubble parameter during inflation, which means the Compton wavelength is large compared to the curvature radius of the de Sitter space $H^{-1}$. So the gravitational effects may play a crucial role on the behavior of the light scalar field in such a scenario. In [20] the authors explicitly showed that the 
quantum fluctuation of the light scalar field $\sigma$ with mass $m$ in de Sitter space gives it a non-zero expectation value of the square of a light scalar field

$$
\left\langle\sigma^{2}\right\rangle=\frac{3 H^{4}}{8 \pi^{2} m^{2}}
$$

where the Hubble parameter $H$ is assumed to be a constant. In [9] we estimated the value of curvaton as $\sigma_{*} \sim H^{2} / m$ and we found $f_{N L}<522 r^{\frac{1}{4}}$.

It is more complicated to estimate the value of curvaton in the models, such as chaotic inflation, where the Hubble constant cannot be regarded as a constant. Fortunately, how to estimate the value of curvaton field in this case has been discussed by Linde and Mukhanov in [21]. According to the long-wave quantum fluctuation of a light scalar field $(m \ll H)$ in inflationary universe, its behavior can be taken as a random walk [22]:

$$
\left\langle\sigma^{2}\right\rangle=\frac{H^{3}}{4 \pi^{2}} t
$$

This equation is only valid for the case of a constant $H$. During inflation the Hubble parameter $H$ is not a constant exactly. We don't explicitly know the behavior of $\left\langle\sigma^{2}\right\rangle$ when $H$ depends on $t$. However, for a short period $\Delta t\left(\ll H^{-1}\right)$, Eq.(17) can be written as

$$
\Delta\left\langle\sigma^{2}\right\rangle \simeq \frac{H^{3}}{4 \pi^{2}}(1-3 \epsilon H \Delta t) \Delta t
$$

Since $3 \epsilon H \Delta t \ll 1$, we suppose that the differential form of Eq.(17)

$$
\frac{d\left\langle\sigma^{2}\right\rangle}{d t} \simeq \frac{H^{3}}{4 \pi^{2}}
$$

can be generalized to the case in which the Hubble parameter slowly varies $\left(\epsilon=-\dot{H} / H^{2} \ll\right.$ 1). On the other hand, a massive scalar field cannot grow up to arbitrary large vacuum expectation value because it has a potential. The long wavelength modes of the light scalar field are in the slow-roll regime and obey the slow-roll equation of motion, i.e.

$$
3 H \frac{d \sigma}{d t}=-\frac{d V(\sigma)}{d \sigma}=-m^{2} \sigma
$$

Combining these two considerations, Linde and Mukhanov proposed in [21]

$$
\frac{d\left\langle\sigma^{2}\right\rangle}{d t}=\frac{H^{3}}{4 \pi^{2}}-\frac{2 m^{2}}{3 H}\left\langle\sigma^{2}\right\rangle
$$

For a constant Hubble parameter, $\left\langle\sigma^{2}\right\rangle$ stabilizes at the point of $\left\langle\sigma^{2}\right\rangle=\frac{3 H^{4}}{8 \pi^{2} m^{2}}$ which is just the same as Eq.(16). Classically the scalar field is stable at $\sigma=0$. In the inflationary 
universe the scalar field $\sigma$ gets a non-zero expectation value due to the gravitational effects. Integrating over Eq.(21) with the initial condition $\left\langle\sigma^{2}\left(t=t_{i}\right)\right\rangle=0$, we obtain

$$
\left\langle\sigma^{2}(t)\right\rangle=\int_{t_{i}}^{t} d t_{1} \frac{H^{3}\left(t_{1}\right)}{4 \pi^{2}} \exp \left(-\int_{t_{1}}^{t} d t_{2} \frac{2 m^{2}}{3 H\left(t_{2}\right)}\right) .
$$

We will use this solution to estimate the value of curvaton field.

Let's take into account the chaotic inflation which is driven by the potential

$$
V(\phi)=\frac{1}{p} \lambda \phi^{p} M_{p}^{4-p}
$$

where $\lambda$ is a small dimensionless parameter $(\lambda \ll 1)$ and $p>0$. From now on we work in the unit of $M_{p}=1$. The equations of motion for the slow-roll inflation are given by

$$
\begin{aligned}
H^{2} & =\frac{\lambda \phi^{p}}{3 p}, \\
3 H \dot{\phi} & =-\lambda \phi^{p-1} .
\end{aligned}
$$

The value of inflaton at the time of $N$ e-folds before the end of inflation is related to $N$ by

$$
\phi_{N}=\sqrt{2 p N}
$$

Now the slow-roll parameter $\epsilon$ becomes

$$
\epsilon=-\frac{\dot{H}}{H^{2}}=\frac{1}{2}\left(\frac{V^{\prime}}{V}\right)^{2}=\frac{p^{2}}{2 \phi_{N}^{2}}=\frac{p}{4 N} .
$$

The number of e-folds corresponds to the CMB scale is roughly $N_{c}=60$. For $n_{s}=0.96$, $p \simeq 4$.8. The amplitude of scalar primordial power spectrum caused by inflaton at CMB scale is

$$
P_{\phi}=\frac{H^{2}}{8 \pi^{2} \epsilon}=\frac{1}{12 \pi^{2} p^{3}} \lambda \phi_{N_{c}}^{p+2} .
$$

In curvaton scenario $P_{\phi} \leq P_{\zeta, \text { obs }}$ which induces an upper bound on $\lambda$, namely

$$
\lambda \leq 12 \pi^{2} p^{3} P_{\zeta, o b s} \phi_{N_{c}}^{-(p+2)} .
$$

For $p=2$ the mass of inflaton $\sqrt{\lambda}$ should be smaller than $6.36 \times 10^{-6}$ in unit of Planck scale. On the other hand, the curvaton mass is smaller than Hubble parameter which says

$$
m^{2} \leq \frac{\lambda \phi^{p}}{3 p} \simeq \frac{\lambda}{3 p}
$$

In the last step we consider that $\phi \sim M_{p}$ at the end of inflation. Combing with Eq.(29), we have

$$
m^{2} \leq 4 \pi^{2} p^{2} P_{\zeta, o b s} \phi_{N_{c}}^{-(p+2)}
$$


For $p=2$, the curvaton mass satisfies $m \leq 2.6 \times 10^{-6}$.

It is time to estimate the vacuum expectation value of curvaton in chaotic inflationary universe. Using the equations of motion for the slow-roll chaotic inflation (24) and (25), we simplify Eq.(21) to be

$$
\left\langle\sigma^{2}(t)\right\rangle \simeq \frac{\lambda}{12 \pi^{2} p^{2}} \int_{\phi(t)}^{\phi_{i}} d \phi_{1} \phi_{1}^{p+1} \exp \left(\frac{2 m^{2}}{\lambda} \int_{\phi_{1}}^{\phi} d \phi_{2} \phi_{2}^{-(p-1)}\right) \simeq \frac{\lambda}{12 \pi^{2} p^{2}(p+2)} \phi_{i}^{p+2},
$$

where we ignore the contribution from the exponential function because its exponent is proportional to $m^{2} / m_{\text {eff }}^{2}(\phi) \sim m^{2} /\left(\lambda \phi^{p-2}\right) \ll 1$. This vacuum expectation value of curvaton mainly comes from the perturbation mode with wavelength $H^{-1}\left(\phi_{i}\right) \exp \left(\frac{\phi_{i}^{2}}{2 p}\right)$. Since the wavelength is much larger than the Hubble horizon, this fluctuation mode is frozen to be a classical one and provides a non-zero classical configuration for curvaton field. The typical value of curvaton field in such a background is estimated as

$$
\sigma_{*}^{2}=\frac{\lambda}{12 \pi^{2} p^{2}(p+2)} \phi_{i}^{p+2}
$$

which is obviously dependent on the initial value of inflaton. Requiring the curvaton energy density be much smaller than inflaton energy density during inflation yields a upper bound on the curvaton mass

$$
m^{2} \ll 24 \pi^{2} p(p+2) \phi_{i}^{-(p+2)} .
$$

Here we also ignore a factor $\phi^{p}$ on the right hand side of the above inequality because $\phi \sim 1$ at the end of inflation. Since the total number of e-folds of chaotic inflation is not very large, the constraint in Eq.(31) is much more stringent than Eq.(34).

In curvaton scenario the primordial power spectrum comes from the quantum fluctuation of curvaton during inflation. Substituting $\sigma_{*}$ in Eq.(33) into (12), we get

$$
P_{\zeta_{\sigma}}=\frac{4 p(p+2)}{9} \Omega_{\sigma, D}^{2} \frac{\phi_{N}^{p}}{\phi_{i}^{p+2}} .
$$

Now the spectral index of primordial power spectrum is given by

$$
n_{s} \equiv 1+\frac{d \ln P_{\zeta_{\sigma}}}{d \ln k} \simeq 1-\frac{d \ln P_{\zeta_{\sigma}}}{d N}=1-\frac{p}{2 N} .
$$

Using Eq.(14), we write down $f_{N L}$ as follows

$$
f_{N L}=\frac{5 \sqrt{p(p+2)}}{6} P_{\zeta, o b s}^{-1 / 2} \frac{\phi_{N}^{p / 2}}{\phi_{i}^{p / 2+1}} .
$$

We need to stress that $f_{N L}$ depends on the initial condition of inflation or the total number of e-folds $N_{t}=\phi_{i}^{2} /(2 p)$ ! For $p=2, f_{N L}=1.84 \times 10^{5} / N_{t}$. If $f_{N L}=100, N_{t}=1.84 \times 10^{3}$. 
The problem is why inflation has such an initial condition. It is very hard to give a physical explanation on it. In this sense, curvaton model can not naturally explain a large non-Gaussian and red tilted primordial power spectrum.

Is it possible that eternal chaotic inflation [23] offers a proper initial condition? During the period of inflation, the evolution of the inflaton field $\phi$ is also influenced by quantum fluctuations, which can also be pictured as a random walk of inflaton with a step $\delta \phi=\frac{H}{2 \pi}$ on a horizon scale per Hubble time $H^{-1}$. During the same epoch, the variation of the classical homogeneous inflaton field rolling down its potential is $\Delta \phi=|\dot{\phi}| \cdot H^{-1}$. Eternal chaotic inflation happens when $\delta \phi=\Delta \phi$, namely

$$
\phi=\phi_{E}=\left(12 \pi^{2} p^{3} / \lambda\right)^{\frac{1}{p+2}}
$$

for the chaotic inflation with potential Eq.(23). Naively we take $\phi_{E}$ as the initial value of inflaton $\phi_{i}$. Therefore we have

$$
\sigma_{*}^{2}=\frac{p}{p+2},
$$

and the primordial power spectrum generated by curvaton

$$
P_{\zeta_{\sigma}}=\frac{p+2}{27 \pi^{2} p^{2}} \lambda \phi_{N_{c}}^{p} \Omega_{\sigma, D}^{2} .
$$

Considering that $\lambda$ is bounded from above by (29) and $P_{\zeta_{\sigma}}=P_{\zeta, \text { obs }}$, we find

$$
\epsilon \geq \frac{9}{8} \Omega_{\sigma, D}^{-2}-\frac{1}{2 N_{c}} .
$$

The slow-roll parameter $\epsilon$ must be larger than one because $\Omega_{\sigma, D} \leq 1$ and the slow-roll condition is violated. The reason is that the energy scale of eternal chaotic inflation is quite high and curvaton gets a large vacuum expectation value which strongly suppresses the amplitude of primordial power spectrum to be smaller than $P_{\zeta, o b s}$. So we conclude that inflation should start at an energy scale lower than eternal inflation scale for the validity of curvaton model.

Before the end of this note, we ignore the initial condition problem and investigate the possible parameter space for curvaton model with a large non-Gaussianity. Following [24], the decay rate of curvaton $\Gamma_{\sigma}$ should be greater than the gravitional-strength decay rate $m^{3}$ in the unit of Planck scale. Combining with Eq.(14) and (15), we obtain

$$
m \leq \frac{2}{15} f_{N L} \sigma_{*}^{2} .
$$

Using Eq.(29), (33) and (37), after a straightforward calculation we find

$$
\sigma_{*}^{2} \leq \frac{25 p}{72 N_{c}} f_{N L}^{-2} .
$$


Combining the above two inequalities leads to an upper bound on the curvaton mass

$$
m \leq 7.7 \cdot 10^{-4} \cdot p / f_{N L} .
$$

If $f_{N L} \sim 100$, this constraint is roughly the same order as Eq.(31). In order to obtain a large non-Gaussianity the curvaton mass should be smaller than $10^{12} \mathrm{GeV}$ in the curvaton model combined with chaotic inflation. On the other hand, the curvaton should decay before neutrino decoupling [24]; otherwise the curvature perturbations may be accompanied by a significant isocurvature neutrino perturbation. The temperature of the universe at the moment of neutrino decoupling is roughly $T_{n d}=1 \mathrm{MeV}$. The curvaton decay rate is bounded by the Hubble parameter at the time of neutrino decoupling from below, i.e. $\Gamma_{\sigma}>\Gamma_{0}=1.68 \times 10^{-43}$ in the unit of $M_{p}=1$. This requirement leads to a loose lower bound on the curvaton mass $m \geq 2.8 \times 10^{-37} f_{N L}^{2} / p^{2}$.

To summarize, the vacuum expectation value of curvaton is sensitive to the physics in the very early universe. In order to get a red-tilted and large non-Gaussian primordial power spectrum in curvaton model we need to choose a suitable initial condition for inflation. As we know, one of the advantage of inflation is that its observational consequences are independent on the initial condition, which make the predictions of inflation strong. Unfortunately the curvaton model we discussed in this note loses this nice point. We need to figure out a more reasonable model with a red-tilted and large non-Gaussian primordial power spectrum in the future.

\section{Acknowledgments}

We would like to thank P. Chingangbam and M. Sasaki for useful discussions. 


\section{References}

[1] A. H. Guth, "The Inflationary Universe: A Possible Solution To The Horizon And Flatness Problems," Phys. Rev. D 23, 347 (1981);

A. D. Linde, "A New Inflationary Universe Scenario: A Possible Solution Of The Horizon, Flatness, Homogeneity, Isotropy And Primordial Monopole Problems," Phys. Lett. B 108, 389 (1982);

A. Albrecht and P. J. Steinhardt, "Cosmology For Grand Unified Theories With Radiatively Induced Symmetry Breaking," Phys. Rev. Lett. 48, 1220 (1982).

[2] A. H. Guth and S. Y. Pi, "Fluctuations In The New Inflationary Universe," Phys. Rev. Lett. 49 (1982) 1110;

V. F. Mukhanov, H. A. Feldman and R. H. Brandenberger, "Theory of cosmological perturbations. Part 1. Classical perturbations. Part 2. Quantum theory of perturbations. Part 3. Extensions," Phys. Rept. 215, 203 (1992).

[3] E. Komatsu et al. [WMAP Collaboration], "Five-Year Wilkinson Microwave Anisotropy Probe (WMAP) Observations:Cosmological Interpretation," arXiv:0803.0547 [astro-ph].

[4] J. M. Maldacena, "Non-Gaussian features of primordial fluctuations in single field inflationary models," JHEP 0305, 013 (2003) arXiv:astro-ph/0210603.

[5] N. Bartolo, E. Komatsu, S. Matarrese and A. Riotto, "Non-Gaussianity from inflation: Theory and observations," Phys. Rept. 402, 103 (2004) arXiv:astro-ph/0406398.

[6] A. P. S. Yadav and B. D. Wandelt, "Detection of primordial non-Gaussianity (fNL) in the WMAP 3-year data at above 99.5\% confidence," arXiv:0712.1148 [astro-ph].

[7] K. Enqvist and M. S. Sloth, "Adiabatic CMB perturbations in pre big bang string cosmology," Nucl. Phys. B 626, 395 (2002) arXiv:hep-ph/0109214.

[8] D. H. Lyth and D. Wands, "Generating the curvature perturbation without an inflaton," Phys. Lett. B 524, 5 (2002) arXiv:hep-ph/0110002;

D. H. Lyth, C. Ungarelli and D. Wands, "The primordial density perturbation in the curvaton scenario," Phys. Rev. D 67, 023503 (2003) arXiv:astro-ph/0208055).

[9] Q. G. Huang, "Large Non-Gaussianity Implication for Curvaton Scenario," arXiv:0801.0467 [hep-th].

[10] K. Ichikawa, T. Suyama, T. Takahashi and M. Yamaguchi, "Non-Gaussianity, Spectral Index and Tensor Modes in Mixed Inflaton and Curvaton Models," arXiv:0802.4138 [astro-ph]. 
[11] T. Multamaki, J. Sainio and I. Vilja, "Non-Gaussianity in three fluid curvaton model," arXiv:0803.2637 [astro-ph].

[12] M. Beltran, "Isocurvature, non-gaussianity and the curvaton model," arXiv:0804.1097 [astro-ph].

[13] X. Chen, M. x. Huang, S. Kachru and G. Shiu, "Observational signatures and non-Gaussianities of general single field inflation," JCAP 0701, 002 (2007) arXiv:hep-th/0605045;

B. Chen, Y. Wang and W. Xue, "Inflationary NonGaussianity from Thermal Fluctuations," arXiv:0712.2345 [hep-th];

M. Li, T. Wang and Y. Wang, "General Single Field Inflation with Large Positive Non-Gaussianity," JCAP 0803, 028 (2008) [arXiv:0801.0040 [astro-ph]];

X. Chen, R. Easther and E. A. Lim, "Generation and Characterization of Large NonGaussianities in Single Field Inflation," JCAP 0804, 010 (2008) arXiv:0801.3295 [astro-ph]];

N. Barnaby and J. M. Cline, "Predictions for Nongaussianity from Nonlocal Inflation," arXiv:0802.3218 [hep-th];

T. Suyama and F. Takahashi, "Non-Gaussianity from Symmetry," arXiv:0804.0425 [astro-ph];

X. Gao, "Primordial Non-Gaussianities of General Multiple Field Inflation," arXiv:0804.1055 [astro-ph];

M. Li, C. Lin, T. Wang and Y. Wang, "Non-Gaussianity, Isocurvature Perturbation, Gravitational Waves and a No-Go Theorem for Isocurvaton," arXiv:0805.1299 [astro$\mathrm{ph}]$;

S. Li, Y. F. Cai and Y. S. Piao, "DBI-Curvaton," arXiv:0806.2363 [hep-ph];

S. W. Li and W. Xue, "Revisiting non-Gaussianity of multiple-field inflation from the field equation," arXiv:0804.0574 [astro-ph];

W. Xue and B. Chen, " $\alpha$-vacuum and inflationary bispectrum," arXiv:0806.4109 [hep-th];

T. Matsuda, "Modulated Inflation," arXiv:0801.2648 [hep-ph];

T. Matsuda, "Running spectral index from shooting-star moduli," JHEP 0802, 099 (2008) [arXiv:0802.3573 [hep-th]].

[14] Q. G. Huang, "Weak gravity conjecture constraints on inflation," JHEP 0705, 096 (2007) arXiv:hep-th/0703071];

Q. G. Huang, "Constraints on the spectral index for the inflation models in string landscape," Phys. Rev. D 76, 061303 (2007) [arXiv:0706.2215 [hep-th]];

Q. G. Huang, "Weak Gravity Conjecture for the Effective Field Theories with N Species," Phys. Rev. D 77, 105029 (2008) arXiv:0712.2859 [hep-th]]. 
[15] X. Chen, S. Sarangi, S. H. Henry Tye and J. Xu, "Is brane inflation eternal?," JCAP 0611, 015 (2006) [arXiv:hep-th/0608082];

D. Baumann and L. McAllister, "A microscopic limit on gravitational waves from D-brane inflation," Phys. Rev. D 75, 123508 (2007) arXiv:hep-th/0610285.

[16] H. Ooguri and C. Vafa, "On the geometry of the string landscape and the swampland," Nucl. Phys. B 766, 21 (2007) arXiv:hep-th/0605264.

[17] L. McAllister and E. Silverstein, "String Cosmology: A Review," Gen. Rel. Grav. 40, 565 (2008) [arXiv:0710.2951 [hep-th]].

[18] Q. G. Huang, "Simplified Chain Inflation," JCAP 0705, 009 (2007) arXiv:0704.2835 [hep-th]];

Q. G. Huang and S. H. Tye, "The Cosmological Constant Problem and Inflation in the String Landscape," arXiv:0803.0663 [hep-th];

D. Chialva and U. H. Danielsson, "Chain inflation revisited," arXiv:0804.2846 [hepth].

[19] E. Silverstein and A. Westphal, "Monodromy in the CMB: Gravity Waves and String Inflation," arXiv:0803.3085 [hep-th].

$[20]$ T. S. Bunch and P. C. W. Davies, "Quantum Field Theory In De Sitter Space: Renormalization By Point Splitting," Proc. Roy. Soc. Lond. A 360 (1978) 117;

A. Vilenkin and L. H. Ford, "Gravitational Effects Upon Cosmological Phase Transitions," Phys. Rev. D 26, 1231 (1982);

A. D. Linde, "Scalar Field Fluctuations In Expanding Universe And The New Inflationary Universe Scenario," Phys. Lett. B 116, 335 (1982).

[21] A. Linde and V. Mukhanov, "The curvaton web," JCAP 0604, 009 (2006) arXiv:astro-ph/0511736.

[22] A. D. Linde, "Particle Physics and Inflationary Cosmology," arXiv:hep-th/0503203.

[23] A. D. Linde, "Eternal Chaotic Inflation," Mod. Phys. Lett. A 1, 81 (1986);

A. D. Linde, "Eternally Existing Selfreproducing Chaotic Inflationary Universe," Phys. Lett. B 175, 395 (1986).

[24] D. H. Lyth, "Can the curvaton paradigm accommodate a low inflation scale," Phys. Lett. B 579, 239 (2004) arXiv:hep-th/0308110. 